\title{
Breast-feeding rates and related maternal and infants' obstetric factors in Japanese twins
}

\author{
Syuichi Ooki
}

Received: 25 November 2007 / Accepted: 16 January 2008/Published online: 22 April 2008

(c) The Japanese Society for Hygiene 2008

\begin{abstract}
Objectives The aims of the present study are to describe the secular trend of breast-feeding rates in Japanese twins in comparison with the general population, and then to clarify the maternal and infants' obstetric factors associated with breast-feeding in twins.

Methods Breast-feeding rates from 0 to 6 full months and related maternal and infant obstetric factors were analyzed using 4,023 Japanese twins, consisting of two different volunteer-based twin samples, age 1-15 years, whose birth year ranged from 1968 to 2003. Data were collected through mailed or hand-delivered questionnaires. Obstetric factors that affect full and partial breast-feeding of twins were confirmed using logistic analyses according to birth order in twin pairs, adjusted by birth year.

Results The full breast-feeding rates of twins were lower than those of the general population. On the other hand, the combined rates of full and partial breast-feeding were close to those of general population, except for the period 19681974. The percentage of concordance pares was around 95\% for all months. The most influential factor that negatively correlated with breast-feeding from 0 to 6 months was gestational weeks. Higher maternal age at twin birth and the use of an incubator also prevented breast-feeding in the neonatal period.

Conclusions The results of the present study for the first time indicated that full breast-feeding of twins has risen recently in Japan, although the rates are still lower than
\end{abstract}

\footnotetext{
S. Ooki $(\square)$

Department of Health Science,

Ishikawa Prefectural Nursing University,

7-1 Tsu, Nakanuma, Kahoku,

Ishikawa 929-1212, Japan

e-mail: sooki@ishikawa-nu.ac.jp
}

those of the general population. The importance of the gestational weeks for breast-feeding rates was also shown.

Keywords Breast-feeding rate - Twin children .

Obstetric factors $\cdot$ Secular trend $\cdot$ Epidemiologic research

\section{Introduction}

Numerous studies show the superiority of human milk for infants. Breast-fed infants show better neurodevelopment and fewer risks of common infections. Epidemiologic studies have revealed a reduced incidence of chronic childhood diseases such as lymphoma, obesity and allergies [1]. Although an adequate quantity and quality of milk production has been documented even for high multiples [2], it seems difficult for mothers rearing multiples to breast-feed for many reasons.

Currently more than $1 \%$ of all births are multiples in Japan. Under such circumstances, there is an increasing need to provide appropriate information to parents and nursing staff, including information on the breast-feeding of multiples. Breast-feeding rates have been examined in Japan every 10 years as part of a national survey on child growth and development conducted by the Ministry of Health, Labor and Welfare [3-6]. In general, many preterm infants or very-low-birthweight infants are reported to be breast-fed less than other infants [7]. If national data are used for health guidance, many twins may be regarded as being breast-fed less. In many cases, professional advisors on child feeding do not have adequate information to answer questions of parents of multiples concerning feeding methods for their children [8]. The reasons many people give for not starting to breast-feed multiples could be avoided if appropriate support is given by family 
members and by the medical team, if they are adequately informed.

There have been many studies concerning the breastfeeding of multiples in Western countries. These reports deal with the introduction of the skills or techniques for breast-feeding multiples [1, 8-14], and practical recommendations or guidelines for the specialist [15-20]. There also exist case reports [21-24], including triplets [25, 26] and quadruplets [18, 27], and detailed descriptions of breast-feeding multiples with relatively small sample sizes $[2,15,25,28,29]$. Some reports [30-38] deal with multiples in comparison with singletons or control groups, or treat multiple births as a risk factor.

In contrast, there is little epidemiologic research $[17,39]$ that focused on breast-feeding multiples. Obstetric factors such as method of delivery, birthweight and gestational period were reported to affect the feeding method of multiples using a relatively small sample size. The aims of the present study are to describe the secular trend of breastfeeding rates in Japanese twins in comparison with the general population, and to clarify the maternal and infants' obstetric factors associated with breast-feeding in twins using the largest database of multiples in Japan.

\section{Methods}

Subjects and outline of data collection

There exist several strategies for the collection of data on multiples in Japan [40]. First, vital statistics can be obtained, but it is almost impossible to obtain access to personal information concerning individuals. Second, data from large hospitals have been used in the field of obstetrics, primarily for the purpose of managing high-risk pregnancies. Third, the Basic Resident Registration of municipalities in Japan can be used. This registration reflects the whole population of each area, and serves as a possible source for recruitment of families with multiples. Nevertheless, this method has many weaknesses. To put it briefly, cost-effectiveness is extremely low in the case of twin studies. Fourth, there is a volunteer-based database of multiples, which includes, for example, data from mothers belonging to associations for parents of multiples. It contains more detailed information on the condition of multiples after birth; both vital statistics and hospital data have difficulty addressing this. Although volunteer-based databases may have some selection biases, cost-effectiveness is very high, if data collection is performed properly.

The present samples used for analysis consisted of volunteer-based Japanese twin databases, involving two independent groups of subjects. The data used in this study were a portion of this body of data. Continuous data on twins in childhood and on their families has been gathered for the purpose of improving twins' health care and of genetic epidemiologic study [41-44]. The first group of subjects consisted of 1,140 mothers and their twin children living in the Tokyo metropolitan area. All the twins in this group had applied to the secondary school attached to the Faculty of Education of the University of Tokyo between 1981 and 2005 (school applicant group). At the time of data collection, all the twins were either 11 or 12 years old (sixth grade of primary school in Japan). Their birth years ranged from 1968 to 1993 [mean 1979, standard deviation (SD) 7 years]. The second group of subjects consisted of 951 mothers and their twin children in several associations for the parents of multiples throughout Japan (maternal association group). The age of the twins during data collection ranged from 1 to 15 years (mean 5.9, SD 3.8). Their birth years ranged from 1986 to 2003 (mean 1995, SD 4 years).

Preliminary analyses and data combination

All data samples were used, irrespective of the data source. Before the data of the two groups were combined, their basic obstetric characteristics were examined in detail [44]. The main results are summarized as follows:

(1) The twins in the maternal association group had 1-week shorter gestations than the twins in the school applicant group.

(2) Body size parameters at birth, namely weight, length, chest circumference, and head circumference were slightly smaller in the maternal association group. Nevertheless, both birth weight itself according to gestational weeks and the percentage difference in relative birth weight within pairs were nearly the same compared to the birth weight norms of the general twin population in Japan [45].

(3) The percentage of mothers treated with ovulationstimulating drugs or in vitro fertilization was much higher in the maternal association group.

These findings suggested that the groups were not very different, at least in their physical development. Moreover, in both groups the data seemed to reflect normal physical development after birth.

Of 2,091 mothers (4,182 twins), 1,140 were from the school applicant group and 951 were from maternal association group. The author excluded 159 twins with no data on feeding method. Birth injuries of mothers or twins, such as placenta previa, placental abruption, coiling of the umbilical cord, neonatal asphyxia, growth-discordant twins, and twin-to-twin transfusion syndrome, were observed to varying degrees. None of these were grounds 
for exclusion from the study. In general, it was very difficult to set clear and consistent inclusion/exclusion criteria, as nearly $50 \%(2,105 / 4,182)$ of the present subjects had at least one of the complications. Moreover, no subjects showed apparent retardation of growth and development at the time of data collection. Thus, 4,023 twins (1,969 males and 2,054 females) aged from 1 to 15 years (mean 9.2 years, SD 3.9 years) of 2,018 mothers were analyzed in this study.

Data were collected through mailed or hand-delivered questionnaires, which had nearly the same format in the two groups. Obstetric and pediatric findings, including feeding methods, were obtained mainly by transcription from the "Maternal and Child Health Handbook." Data based mainly on mass examinations are recorded in this handbook, and it serves as a source of health information for pregnant women, as it contains detailed medical records on pregnancy and delivery, as well as on child care, for children up to 6 years old [46, 47]. The mothers were advised to refer to those records when completing the questionnaire. If mothers could not remember events relating to their children, the author recommended that, in the questionnaire, they should not use incorrect answers merely to complete the questionnaire, but give a blank response. The zygosity of the twins was determined primarily from a questionnaire [48]. For the school applicant group, zygosity was diagnosed also by the use of many genetic markers for those twin pairs who were actually admitted to school [48].

These questionnaire surveys are now in progress. The response rates of the questionnaire were $100 \%$ for the school applicant group and more than $70 \%$ for the maternal association group.

\section{Questions concerning breast-feeding}

Feeding methods were divided into three categories: full breast-feeding, partial breast-feeding (mixed feeding) and formula feeding. The detailed method of feeding and the percentage of breast milk consumed in partial breastfeeding were not obtained. Mothers recorded the duration (starting and ending full month) of each feeding method. The data were organized according to the full month. Therefore, the first 30 days represented 0 months.

\section{Statistical analyses}

First, breast-feeding rates were calculated from 0 to 6 full months. To compare the results with the official Japanese data on breast-feeding rates of 1-4 full months (1970, 1980, 1990 and 2000 surveys), the birth years of the twins were divided into four groups: 1968-1974, 1975-1984, 1985-1994 and 1995-2003. The first two birth year groups contained only members of the school applicant group, and the fourth group contained only members of the maternal association group. The third group contained 536 twins from the school applicant group and 694 twins from the maternal association group.

Next, within-pair differences in feeding method were analyzed according to age in months, using twin pairs with both twins' complete data on frequency.

Finally, maternal and infants' obstetric factors that affected the selection of the kind of breast-feeding of twins were confirmed. Following the previous guidelines for breast-feeding multiples [10, 16, 18] and possible low rate of full breast-feeding, the author regarded full breastfeeding and partial breast-feeding in one category as "at least some breast milk feeding" [30, 31, 49] in the following analyses. Sixteen variables with a less than $3 \%$ frequency of missing values were analyzed. The quantitative variables considered were: birth year of twins, gestational age (weeks), birthweight (g), maternal age at twin birth, and the number of birth injuries if any. The quantitative variables were dichotomized in accordance with the results of a preliminary analysis of the effects of a single variable on the selection of breast-feeding. The qualitative variables considered were: sex (male or female), parity (primipara or multipara), zygosity (monozygotic or dizygotic), presentation (vertex or non-vertex), placentation (monochorionic or dichorionic), method of delivery (Caesarean section or not), fertility treatment (yes or no), use of incubator (yes or no), previous abortion (yes or no), health condition at birth (neonatal asphyxia or not), and subject groups (school applicant group or maternal association group). All variables were then transformed into qualitative variables, and the codes of 0 or 1 were given.

Logistic regression analysis was performed with a threshold significance level of 0.05 according to the birth order of twins (first-born and second-born) to avoid overestimation based on the independence of the sib-pair data. All statistical analyses were performed using $\mathrm{SAS}^{\circledR}$ for Windows [50].

\section{Ethical issues}

The methods of informed consent vary according to the subjects. As to school applicant group, the statistical analysis of the data was clearly written in the application document, and the detailed explanations concerning data collection by questionnaire and interview, and blood sampling for zygosity examination and health check were added as another paper from 1999. Moreover, informed 
consent was obtained from each twin and his or her parents in writing from 2001 on as part of the application process. The data analysis was also permitted by the twin research committee of this school. When present study was performed, this school did not have an ethical committee for the twin study, which is now under construction, including the author as one of the advisers. Zygosity diagnosis using DNA sample was permitted through the ethical committee of the Graduate School of Medicine, University of Tokyo [51].

All the mothers in the maternal association group cooperated voluntarily in this research, mainly through the presidents of their associations [51].

These twin studies were also permitted by the ethical committee of the Ishikawa Prefectural Nursing University in 2004.

\section{Results}

The breast-feeding rates according to birth year groups are shown in Table 1. The breast-feeding rate at 0 months was about $10 \%$ in 1968-1974, and around 20\% in 1975-1984, 1985-1994, and 1995-2003. In the 1985-1994 birth year period, which contained twins from both the school applicant and maternal association groups, there was no significant difference of feeding method between the two groups for any month (data not shown).

The breast-feeding rates were compared to those reported in Japanese national surveys. The results are shown in Fig. 1. The full breast-feeding rates of twins were lower than that of the general population. On the other hand, the combined rates of full and partial breast-feeding were close to those of the general population, except in the 1968-1974 periods.

Within-pair differences of the feeding method are shown in Table 2. The percentage of concordance pares were around $95 \%$ in all months. Complete discordance, namely when one twin is breast-fed and the other twin is formulafed, was seen in about $1 \%$ of cases in each month. Concordance rates and polychoric correlations were constantly higher in monozygotic pairs than dizygotic pairs (data not shown).

As the effect of birth year was thought to be large, as shown in Table 1 and Fig. 1, this variable was checked carefully prior to main analyses. As was expected, the birth year effect was very strong. Therefore, the author always adjusted for birth year in the logistic analyses.

Birth year was dichotomized in two patterns, 1968-1974 vs $1975-2003$ or 1968-1984 vs 1985-2003, and logistic analyses were performed according to these two patterns of birth year classification. As the latter classification (19681984 vs 1985-2003) showed a slightly clearer effect on the breast-feeding rates of each obstetric variable than the former (1968-1974 vs 1975-2003), the results of the latter classification were used in Tables 3 and 4.

The results of the univariate logistic analyses adjusted by birth year are shown in Table 3. Gestational age, birthweight, maternal age and use of incubator had significant effects on both first- and second-born twins at 0 months. The effect of the gestational age remained significant at 3 and 6 months. No effect of subject groups was observed.

Multivariate logistic analyses were performed using all significant variables in each of the univariate analyses. The results are shown in Table 4 . Gestational weeks for firstborn twins, maternal age and use of incubator for both first- and second-born twins were selected at 0 months, gestational weeks for first-born twins, maternal age and use of incubator for second-born twins was selected at 3 months, and only gestational weeks for both first- and second-born twins was selected at 6 months.

\section{Discussion}

\section{Breast-feeding rates of twins}

Since the report of Addy [52], many other reports [15, 30, $31,36,38,49,53-57]$ have been completed, with a variety of levels of reliability and verifiability. Although reports from a Western maternal association group [17, 39] contained a large sample size, detailed statistic analyses of the data were not performed. To the author's knowledge, the present study is one of the largest twin studies that includes an analysis of secular trends of breast-feeding twins with detailed information on monthly feeding method and obstetric findings for mothers and twins.

The breast-feeding rates of multiples vary considerably in the literature. This wide variation is probably due as much to differences in the definitions of breast-feeding (strictly exclusive breast-feeding or roughly at least some breast-feeding), the methods of collecting data (retrospective questionnaire, medical record or interview), and the sampling population (a highly motivated group, mothers treated with medical intervention or general population of multiples). In addition, cultural differences may be very large. Breast-feeding rates are nearly $100 \%$, for example, in southern and eastern Africa, irrespective of the plurality of birth [32].

In the present study, a simple questionnaire was used to obtain retrospective data. The questions concerning feeding method seemed not so different from those used in the national survey in Japan [3-6] or in little epidemiologic studies of feeding method of multiples in other countries [17, 39]. 
Table 1 Percentage of feeding type of twin individuals according to the birth year of twins

\begin{tabular}{|c|c|c|c|c|c|c|c|}
\hline & \multicolumn{2}{|c|}{ Full breast-feeding } & \multicolumn{2}{|c|}{ Partial breast-feeding } & \multicolumn{2}{|c|}{ Formula feeding } & \multirow{2}{*}{$\begin{array}{l}\text { Missing } \\
n\end{array}$} \\
\hline & $n$ & $\%$ & $n$ & $\%$ & $n$ & $\%$ & \\
\hline \multicolumn{8}{|c|}{$1968-1974(n=729)$} \\
\hline 0 months & 83 & 11.8 & 285 & 40.5 & 335 & 47.7 & 26 \\
\hline 1 month & 59 & 9.7 & 230 & 38.0 & 317 & 52.3 & 123 \\
\hline 2 months & 50 & 8.7 & 181 & 31.5 & 344 & 59.8 & 154 \\
\hline 3 months & 35 & 6.1 & 173 & 30.1 & 366 & 63.8 & 155 \\
\hline 4 months & 21 & 3.8 & 146 & 26.1 & 392 & 70.1 & 170 \\
\hline 5 months & 17 & 3.2 & 128 & 24.2 & 384 & 72.6 & 200 \\
\hline 6 months & 11 & 2.1 & 120 & 23.2 & 386 & 74.7 & 212 \\
\hline \multicolumn{8}{|c|}{ 1975-1984 $(n=935)$} \\
\hline 0 months & 177 & 19.5 & 531 & 58.4 & 202 & 22.2 & 25 \\
\hline 1 month & 143 & 16.8 & 480 & 56.3 & 230 & 27.0 & 82 \\
\hline 2 months & 133 & 16.2 & 374 & 45.5 & 315 & 38.3 & 113 \\
\hline 3 months & 100 & 12.4 & 365 & 45.3 & 341 & 42.3 & 129 \\
\hline 4 months & 54 & 6.8 & 321 & 40.3 & 421 & 52.9 & 139 \\
\hline 5 months & 49 & 6.4 & 276 & 36.2 & 438 & 57.4 & 172 \\
\hline 6 months & 43 & 5.8 & 257 & 34.9 & 436 & 59.2 & 199 \\
\hline \multicolumn{8}{|c|}{ 1985-1994 $(n=1,230)$} \\
\hline 0 months & 253 & 21.1 & 813 & 67.9 & 132 & 11.0 & 32 \\
\hline 1 month & 194 & 16.8 & 802 & 69.3 & 161 & 13.9 & 73 \\
\hline 2 months & 190 & 16.8 & 668 & 59.0 & 274 & 24.2 & 98 \\
\hline 3 months & 161 & 14.4 & 604 & 53.9 & 355 & 31.7 & 110 \\
\hline 4 months & 121 & 11.2 & 502 & 46.4 & 459 & 42.4 & 148 \\
\hline 5 months & 100 & 9.4 & 476 & 44.8 & 487 & 45.8 & 167 \\
\hline 6 months & 92 & 8.8 & 460 & 43.7 & 500 & 47.5 & 178 \\
\hline \multicolumn{8}{|c|}{ 1995-2003 $(n=1,129)$} \\
\hline 0 months & 222 & 20.0 & 735 & 66.1 & 155 & 13.9 & 17 \\
\hline 1 month & 180 & 16.7 & 739 & 68.6 & 159 & 14.8 & 51 \\
\hline 2 months & 169 & 15.9 & 634 & 59.5 & 262 & 24.6 & 64 \\
\hline 3 months & 148 & 14.0 & 586 & 55.2 & 327 & 30.8 & 68 \\
\hline 4 months & 115 & 11.0 & 489 & 46.8 & 440 & 42.2 & 85 \\
\hline 5 months & 113 & 11.0 & 432 & 41.9 & 487 & 47.2 & 97 \\
\hline 6 months & 106 & 10.5 & 394 & 39.1 & 508 & 50.4 & 121 \\
\hline
\end{tabular}

In the present sample in the 1995-2003 periods, full breast-feeding rates were $20.0 \%$ and partial breast-feeding rates were $86.1 \%$ at 0 months postpartum (Table 1), reflecting the importance of the definition of breast-feeding. As shown in Fig. 1, compared with the Japanese general population, the present subjects showed a lower rate of full breast-feeding. On the other hand, the combined rates of full and partial breast-feeding were close to those of the general population except in the 1968-1974 periods. The breast-feeding rates of twins clearly rose after the 1975 period. The most likely reason for this is that a national campaign of breast-feeding promotion was started in 1975 in Japan, followed by the WHO recommendation on breastfeeding.
Mothers of multiples achieve very high breast-feeding initiation rates as shown in studies of twins' mothers clubs, with rates of $70-90 \%[10,12,17,56,57]$. This range, however, may well represent the rates that can be obtained in a highly motivated select group, and demonstrates the importance of support groups [1].

With regard to whether the breast-feeding rates between singletons and twins differ, several studies have been performed, and conflicting evidence has been reported [1, 8]. According to Addy [52], the breast-feeding rates of twins (23.7\%, $n=173$ mothers) are similar to those of general singletons. Neifert and Thorpe [12] quoted the Ross Quarterly Mothers' survey of 1988, which showed that mothers of twins were less likely either to initiate breast-feeding in 
the hospital or to continue nursing at 6 months postpartum when compared with all mothers. Wilton [39] quoted Ross Laboratories National Mothers' Surveys (unpublished data)
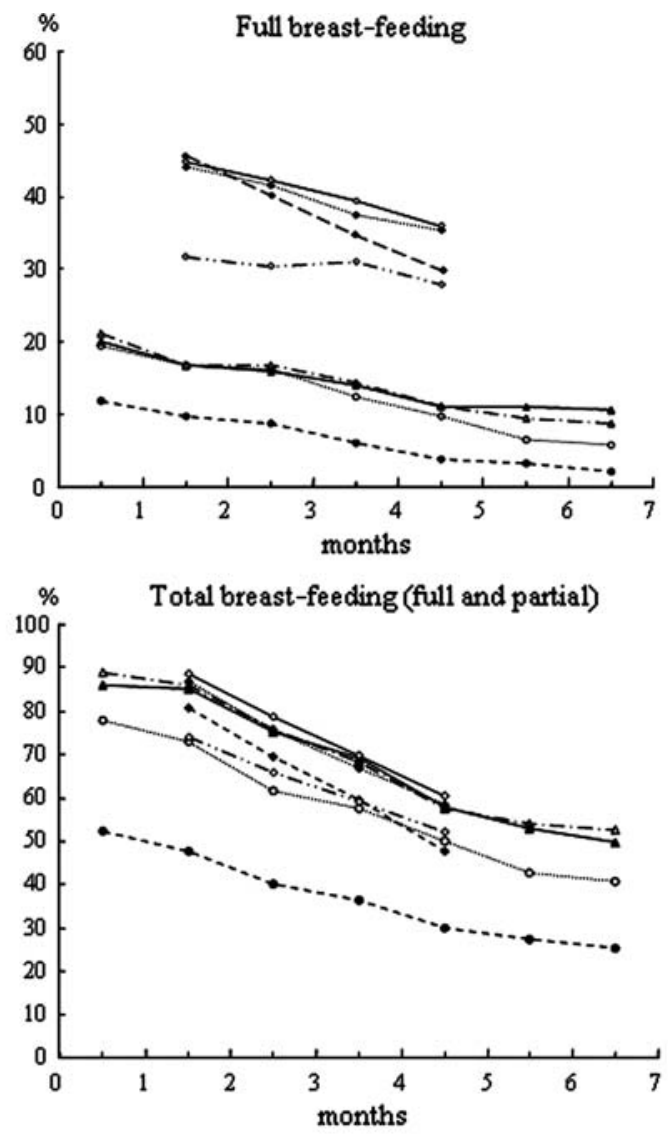

\begin{tabular}{|lll|}
\hline Twin data & $-\bullet 1968-1974(n=729)$ & $-0-1975-1984(n=939)$ \\
& $-\Delta-1985-1994(n=1230)$ & $\leftarrow 1995-2003(n=1129)$ \\
National survey & $-\diamond-1970$ & $-\leftarrow 1980$ \\
& -1990 & $-\diamond 2000$ \\
\hline
\end{tabular}

Fig. 1 Breast-feeding rates of twins according to birth year group and reported that the initiation of breast-feeding was $54 \%$ in singletons and $48 \%$ in twins, decreasing to $20 \%$ and $13 \%$ respectively at 6 months. Population-based statistics in Wales in 2004 showed that the initiation rate of breastfeeding was $52 \%$ for singletons, $40 \%$ for twins, and $15 \%$ for triples [1].

Epidemiologic studies that have included twins and/or high order multiples other than singletons have consistently shown that twins/multiples are significantly less breast-fed exclusively at discharge and at 4 weeks of age [53], at 23 months postpartum [37] and throughout early childhood [54]. According to the Japanese National Survey "First Longitudinal Survey of Babies in the 21st Century", exclusive breast-feeding rate during first 6 months was $21.5 \%$ in singletons $(n=45,594)$ and $1.3 \%$ in multiples $(n=975)$. Of many factors associated with exclusive breast-feeding, multiple births were the strongest negative factor for exclusive breast-feeding [33].

There exist several hospital-based intervention studies with relatively small sample size (less than 100 mothers). Despite an intensive promotion program, it was rare for multiples to be discharged on exclusive breast-feeding, although $90-100 \%$ of women $(n=22$ twins [15] or $n=60$ twin pairs [55]) provided at least some breast milk at the time of discharge from the hospital. Liang et al. [36] reported that $89 \%$ of preterm twins $(n=18)$ and $93 \%$ of preterm singletons $(n=15)$ were breast-feeding with or without additional bottle feedings when discharged from the hospital. Flidel-Rimon and Shinwell [58] showed that a dramatic increase in breast-feeding rates in very low birthweight singletons and twins has been noted in recent years (1995-2002), due in no small part to appropriate hospital policies and practices. These reports suggest that if partial breast-feeding was regarded as a success, twins can be breast-fed as successfully as singletons with sufficient assistance and encouragement.

Table 2 Feeding method of twin pairs

\begin{tabular}{|c|c|c|c|c|c|c|c|c|c|c|c|c|}
\hline & \multicolumn{6}{|c|}{ Concordant } & \multicolumn{6}{|c|}{ Discordant } \\
\hline & \multicolumn{2}{|l|}{$\begin{array}{l}\text { Full } \\
\text { breast- } \\
\text { feeding }\end{array}$} & \multicolumn{2}{|l|}{$\begin{array}{l}\text { Partial } \\
\text { breast- } \\
\text { feeding }\end{array}$} & \multicolumn{2}{|c|}{ Formula feeding } & \multicolumn{2}{|c|}{$\begin{array}{l}\text { Full breast- } \\
\text { feeding } \\
\text { and partial } \\
\text { breast-feeding }\end{array}$} & \multicolumn{2}{|c|}{$\begin{array}{l}\text { Partial breast- } \\
\text { feeding and } \\
\text { formula } \\
\text { feeding }\end{array}$} & \multicolumn{2}{|c|}{$\begin{array}{l}\text { Full breast- } \\
\text { feeding } \\
\text { and formula } \\
\text { feeding }\end{array}$} \\
\hline & $n$ (pairs) & $\%$ & $n$ (pairs) & $\%$ & $n$ (pairs) & $\%$ & $n$ (pairs) & $\%$ & $n$ (pairs) & $\%$ & $n$ (pairs) & $\%$ \\
\hline 0 months & 339 & 17.4 & 1,137 & 58.3 & 380 & 19.5 & 39 & 2.0 & 42 & 2.2 & 14 & 0.7 \\
\hline 1 month & 267 & 14.5 & 1,092 & 59.4 & 407 & 22.2 & 25 & 1.4 & 32 & 1.7 & 14 & 0.8 \\
\hline 2 months & 246 & 13.8 & 899 & 50.3 & 575 & 32.1 & 29 & 1.6 & 23 & 1.3 & 17 & 1.0 \\
\hline 3 months & 201 & 11.3 & 839 & 47.3 & 674 & 38.0 & 25 & 1.4 & 21 & 1.2 & 15 & 0.8 \\
\hline 4 months & 137 & 7.9 & 706 & 40.7 & 836 & 48.2 & 20 & 1.2 & 21 & 1.2 & 14 & 0.8 \\
\hline 5 months & 121 & 7.2 & 635 & 37.6 & 874 & 51.8 & 16 & 0.9 & 24 & 1.4 & 17 & 1.0 \\
\hline 6 months & 108 & 6.6 & 595 & 36.1 & 893 & 54.1 & 14 & 0.8 & 24 & 1.5 & 16 & 1.0 \\
\hline
\end{tabular}




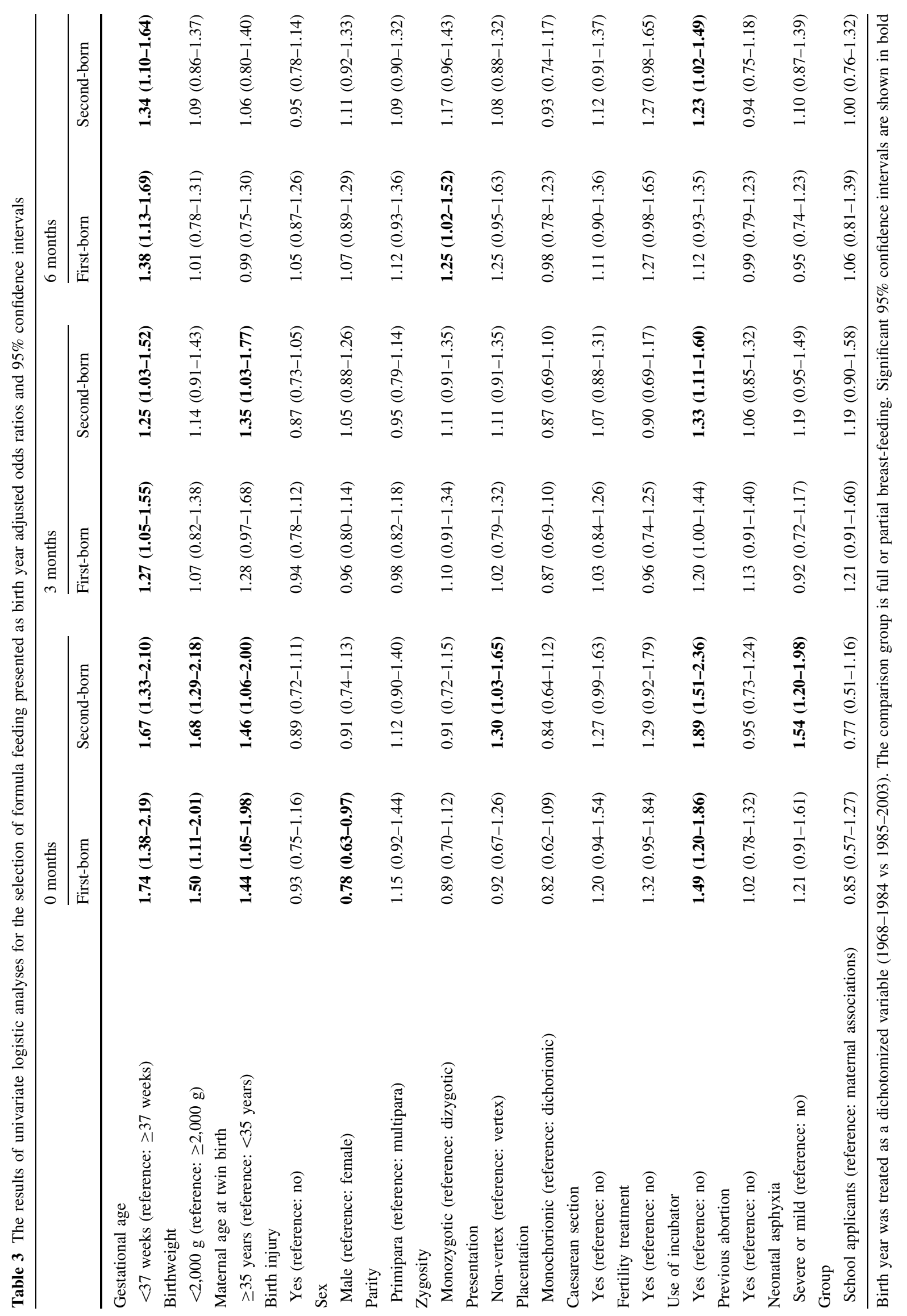


Table 4 The results of multivariate logistic analyses for selection of formula feeding presented as odds ratios and $95 \%$ confidence intervals

\begin{tabular}{|c|c|c|c|c|c|c|c|}
\hline & & \multicolumn{2}{|l|}{0 months } & \multicolumn{2}{|l|}{3 months } & \multicolumn{2}{|l|}{6 months } \\
\hline & & First-born & Second-born & First-born & Second-born & First-born & Second-born \\
\hline Birth year & $\begin{array}{l}\text { 1968-1984 } \\
\quad \text { (reference: } \\
\text { 1985-2003) }\end{array}$ & $3.29(2.57-4.21)$ & $3.79(2.96-4.86)$ & $2.61(2.16-3.14)$ & $2.70(2.23-3.27)$ & $2.24(1.82-2.76)$ & $2.30(1.87-2.82)$ \\
\hline Birthweight & $\begin{array}{l}<2,000 \mathrm{~g} \\
\quad \text { (reference: } \\
\quad \geq 2,000 \mathrm{~g} \text { ) }\end{array}$ & Not selected & Not selected & - & - & - & - \\
\hline $\begin{array}{l}\text { Maternal } \\
\text { age }\end{array}$ & $\begin{array}{l}\geq 35 \text { years } \\
\quad \text { (reference: } \\
\quad<35 \text { years) }\end{array}$ & $1.54(1.08-2.20)$ & $1.51(1.05-2.15)$ & - & $1.34(1.02-1.77)$ & - & - \\
\hline $\begin{array}{l}\text { Gestational } \\
\text { age }\end{array}$ & $\begin{array}{l}<37 \text { weeks } \\
\quad \text { (reference: } \\
\quad \geq 37 \text { weeks) }\end{array}$ & $1.50(1.14-1.97)$ & Not selected & $1.28(1.06-1.56)$ & Not selected & $1.36(1.10-1.67)$ & $1.30(1.06-1.61)$ \\
\hline Sex & $\begin{array}{l}\text { Male } \\
\text { (reference: } \\
\text { female) }\end{array}$ & not selected & - & - & - & - & - \\
\hline Zygosity & $\begin{array}{r}\text { Monozygotic } \\
\text { (reference: } \\
\text { dizygotic) }\end{array}$ & - & - & - & - & Not selected & - \\
\hline Presentation & $\begin{array}{l}\text { Non-vertex } \\
\text { (reference: } \\
\text { vertex) }\end{array}$ & - & Not selected & - & - & - & - \\
\hline $\begin{array}{l}\text { Use of } \\
\text { incubator }\end{array}$ & $\begin{array}{l}\text { Yes } \\
\text { (reference: } \\
\text { no) }\end{array}$ & $1.34(1.03-1.74)$ & $1.92(1.50-2.46)$ & - & $1.35(1.12-1.62)$ & - & Not selected \\
\hline $\begin{array}{l}\text { Neonatal } \\
\text { asphyxia }\end{array}$ & $\begin{array}{l}\text { Severe or mild } \\
\text { (reference: } \\
\text { no) }\end{array}$ & - & Not selected & - & - & - & - \\
\hline
\end{tabular}

The comparison group is full or partial breast-feeding

The present study showed that the feeding method was generally similar in both twins, but about $5 \%$ of the twin pairs were reared on different feeding methods from birth to 6 months. Most reports on the breast-feeding of multiples did not consider the differences between individual twins. According to Gromada and Spangler [10], monozygotic pairs were more similar than dizygotic pairs in terms of their styles of breast-feeding, including the length of feedings and time between feedings, which the mothers of twins surely recognize. The present results showed that monozygotic pairs were more similar than dizygotic pairs as to feeding method (data not shown), partly supporting the above indication. As for the proportion of breast milk fed to each sibling in a pair of twins, there was almost complete agreement in the proportion of breast milk fed to sibling infant twins [49]. The present results are in accordance with this.

Factors affecting the breast-feeding of twins

There exist many reports that examine the factors that interfere with the breast-feeding of multiples, with relatively small sample size. Difficulties with breast-feeding multiples include insufficient prenatal and early postpartum breast-feeding education and support, delayed lactogenesis, insufficient milk supply, problems with latch and positioning, profound maternal fatigue, and parental mental health issues $[1,8,10,15,16,19,25,30,58]$. Prematurity often interferes with breast-feeding [28]. More general factors, such as education level, income and smoking were often identified [7, 37].

The present study focused mainly on maternal and infants' perinatal and neonatal factors, and represents the first attempt to analyze the factors related to breast-feeding twins at the individual twin level. Geraghty et al. [49] pointed out the essential difference in the definition of breast-feeding between multiples and singletons. Current methods of obtaining breast-feeding data for the mothers of singletons may not adequately describe the breast-feeding behaviors of multiples, because most data do not consider the difference within the pair.

The results of logistic analysis showed that the effect of gestational age on breast-feeding rates was consistently the largest when the birth year effect was adjusted. The effect 
of birthweight was also significant for univariate analyses, especially at 0 months. This effect was not found in the multivariate analyses. As birthweight is strongly correlated with gestational age, the effect of birthweight was thought to be weakened. In singletons, birthweight and prematurity are recognized risk factors for breast-feeding inhibition. The effect of gestational age or prematurity on the breastfeeding rates of twins has been pointed out several times $[8,30]$.

The effect of maternal age at twin birth and the use of an incubator on breast-feeding rates at 0 months were observed. This effect was partly observed at 3 and 6 months. In this case, older mothers and those of twins where an incubator was used tended to breast-feed less. The effect of maternal age and use of an incubator tended to be greater on second-born twins. It was unclear why these effects were stronger in second-born twins. The frequency of incubator use was higher in second-born twins, partly reflecting the high-risk status of second-born twins in general. The effect of sex, presentation, zygosity and neonatal asphyxia, which was seen in univariate analyses, was not observed in multivariate analyses. All previous studies treated breast-feeding multiples only as a matter of differences among mothers, and did not consider the difference of individual twins. The present results suggested that inhibiting factors of breast-feeding multiples may in part differ according to birth order in twins.

The effect of birth injury, method of delivery (Caesarean section or not) and health condition at birth (neonatal asphyxia or not) are very complicated with respect to twin delivery. More than one type of birth injury was observed in about half of the present subjects, with various levels of severity. At the population level, this effect was weakened, confounded with many other variables. At the case-study level, neonates with severe birth injury may well be less breast-fed. For example, breast-feeding multiples after a Caesarean section is not a simple task [8].

Moreover, maternal mental condition or stress is thought to affect breast-feeding rates [35]. Feeding infants was found to be significantly more stressful for mothers of twins than singletons [59]. Socioeconomic factors are also likely to be important in breast-feeding [7, 37]. Some of these risk factors have been more frequently observed in twin pregnancies and nurturing.

In addition, maternal affect and/or coping attributes were likely to differ depending upon whether singletons or multiples were involved [35]. There is a possibility that social and psychological factors-for example prenatal education for breast-feeding, mental condition or anxiety, and support from families and medical staff-are more important with respect to breast-feeding multiples than singletons. The present results suggested that more detailed study should be performed [17], including epidemiologic study of breast-feeding multiples at the individual infant level.

\section{Limitations}

The definition of breast-feeding in this study was very simple, as were the questions used to collect data on the total growth and development of twins. The definition of breast-feeding has been discussed several times, and strict definitions also exist [56, 57, 60, 61]. Some authors [10, 30, 31, 49] use the terminology human/breast milk feeding, instead of breast-feeding, since it is common practice for preterm infants to be fed expressed or pumped human milk.

In addition, the present data were limited to normally developed twins, and consequently may have had a positive selection bias, especially for socioeconomic factors, which would raise the breast-feeding rate. There was no positive selection bias in the present samples, at least in the normal range of birthweight [44], compared with twins' birthweight norms according to gestational age, as indicated by Japanese vital statistics [45], mentioned in the section "Preliminary analyses and data combination".

The merits and demerits of combining the data of the two subject groups should be discussed. One reason why the author combined the data was that the groups were of similar size. When discussing the secular trend of breastfeeding rates in the present study, readers should remember that the 1968-1974, 1975-1984 groups contained only members of the school applicant group, and the 1995-2003 groups contained only members of the maternal association group. To analyze a trend-taking place over more than 30 years, the combination of the two different samples is the second-best possible method. Moreover, although birth year was adjusted in all logistic regression analyses, and group difference was not a significant factor on breastfeeding rates, some unexpected selection biases or confounding may have occurred. These effects could be estimated by comparing the data to less biased, more representative, and nationwide data on twins, which was almost impossible to obtain in Japan.

Given the study design, there was a potential for recall bias. The author considered that in the questionnaire survey there was no method of obtaining breast-feeding data from a large number of mothers at the same time and with relatively the same accuracy, other than by using the "Maternal and Child Health Handbook", as this booklet is the basic reference regarding maternal and child health care in Japan. Most of the important records and information about children's development are recorded in this handbook. It is possible that the older the twins became, the more unreliable the memory of their mother became at data collection time, particularly if she depended only on her 
memory. These methodological faults cannot be adjusted for, and represent the largest limitation of the present questionnaire survey. Nevertheless, there have been many studies that have cited maternal recall of perinatal and infant feeding events as being accurate years later [62-65].

\section{Future direction and conclusion}

The results of the present study indicated that the rate of full breast-feeding of twins has risen recently in Japan, although it is still much lower than that of the general population, if the data gathered about the monthly feeding method after birth is correct. Combined rates of full and partial breast-feeding were close to those of the general population. The most influential factor that negatively correlated with breast-feeding from 0 to 6 months was gestational weeks. Higher maternal age at twin birth and the use of an incubator also reduced the level of breastfeeding in the neonatal period.

Antenatal counseling [17], hospital practice, the attitude of the medical team towards breast-feeding [58], the expertise of the public health nurse, and national policies on maternal and child health could change the current situation. However, when breast-feeding is not possible, the health caregiver must carefully avoid judgmental approaches that may induce guilt [8], and mothers should not be given the impression that they have to breast-feed exclusively in order to breast-feed successfully [17, 18]. The present results showed that it was desirable to raise the full breast-feeding rates of multiples while maintaining the total (full and partial) breast-feeding rates by following the above guidelines. Further research on the uniqueness and variability of breast-feeding multiples [17] can lead to the development of practices that increase breast-feeding rates for multiples in Japan.

Acknowledgments The author gratefully acknowledges the help of Toshimi Ooma in the analysis of the data and the help of Dr. Noriko Kato of the Japanese National Institute of Public Health for useful suggestion and information.

\section{References}

1. Flidel-Rimon O, Shinwell ES. Breast feeding twins and high multiples. Arch Dis Child Fetal Neonatal Ed. 2006;91:F377-80.

2. McKenzie PJ. The seeking of baby-feeding information by Canadian women pregnant with twins. Midwifery. 2006;22:21827.

3. Nihon syouni hoken kyoukai. Growth standards of Japanese young children. Research by Ministry of Health and Welfare of Japan in 1970. Tokyo: Nihon syouni hoken kyoukai; 1972 (in Japanese).

4. Nihon syouni hoken kyoukai. Growth standards of Japanese young children. Research by Ministry of Health and Welfare of
Japan in 1980. Tokyo: Nihon syouni hoken kyoukai; 1982 (in Japanese).

5. Nihon syouni hoken kyoukai. Growth standards of Japanese young children. Research by Ministry of Health and Welfare of Japan in 1990. Tokyo: Nihon syouni hoken kyoukai; 1992 (in Japanese).

6. Nihon syouni hoken kyoukai. Growth standards of Japanese young children. Research by Ministry of Health and Welfare of Japan in 2000. Tokyo: Nihon syouni hoken kyoukai; 2002 (in Japanese).

7. Killersreiter B, Grimmer I, Buhrer C, Dudenhausen JW, Obladen M. Early cessation of breast milk feeding in very low birthweight infants. Early Hum Dev. 2001;60:193-205.

8. Flidel-Rimon O, Shinwell ES. Breast-feeding multiples. Semin Neonatol. 2002;7:231-9.

9. Bryan E. Twins in the family. Nurs Times. 1983;79:50-2.

10. Gromada KK, Spangler AK. Breastfeeding twins and higherorder multiples. J Obstet Gynecol Neonatal Nurs. 1998;27:441-9.

11. Leonard LG. Breastfeeding twins: maternal-infant nutrition. JOGN Nurs. 1982;11:148-53.

12. Neifert M, Thorpe J. Twins: family adjustment, parenting, and infant feeding in the fourth trimester. Clin Obstet Gynecol. 1990;33:102-13.

13. Stables J. Breastfeeding twins. Nurs Times. 1980;76:1493-4.

14. Walker M. Breastfeeding quadruplets. J Obstet Gynecol Neonatal Nurs. 1993;22:15.

15. Hattori R, Hattori H. Breastfeeding twins: guidelines for success. Birth. 1999;26:37-42.

16. Leonard LG. Breastfeeding higher order multiples: enhancing support during the postpartum hospitalization period. J Hum Lact. 2002;18:386-92.

17. Leonard LG. Breastfeeding rights of multiple birth families and guidelines for health professionals. Twin Res. 2003;6:34-45.

18. Mead LJ, Chuffo R, Lawlor-Klean P, Meier PP. Breastfeeding success with preterm quadruplets. J Obstet Gynecol Neonatal Nurs. 1992;21:221-7.

19. Sollid DT, Evans BT, McClowry SG, Garrett A. Breastfeeding multiples. J Perinat Neonatal Nurs. 1989;3:46-65.

20. Spiro A. Supporting parents who wish to breastfeed twins. Health Visit. 1992;65:197-8.

21. Biancuzzo M. Breastfeeding preterm twins: a case report. Birth. 1994;21:96-100.

22. Emsley A. Breast-feeding twins. Midwives Chron. 1994;107:178-81.

23. LaFleur EA, Niesen KM. Breastfeeding conjoined twins. J Obstet Gynecol Neonatal Nurs. 1996;25:241-4.

24. Schecter A, Papke O, Lis A, Ball M, Ryan JJ, Olson JR, et al. Decrease in milk and blood dioxin levels over two years in a mother nursing twins: estimates of decreased maternal and increased infant dioxin body burden from nursing. Chemosphere. 1996;32:543-9.

25. Leonard LG. Breastfeeding triplets: the at-home experience. Public Health Nurs. 2000;17:211-21.

26. Storr GB. Breastfeeding premature triplets: one woman's experience. J Hum Lact. 1989;5:74-7.

27. Auer C, Gromada KK. A case report of breastfeeding quadruplets: factors perceived as affecting breastfeeding. J Hum Lact. 1998;14:135-41.

28. Nyqvist KH. Breast-feeding in preterm twins: development of feeding behavior and milk intake during hospital stay and related caregiving practices. J Pediatr Nurs. 2002;17:246-56.

29. Saint L, Maggiore P, Hartmann PE. Yield and nutrient content of milk in eight women breast-feeding twins and one woman breastfeeding triplets. Br J Nutr. 1986;56:49-58.

30. Geraghty SR, Pinney SM, Sethuraman G, Roy-Chaudhury A, Kalkwarf HJ. Breast milk feeding rates of mothers of multiples 
compared to mothers of singletons. Ambul Pediatr. 2004;4:22631.

31. Geraghty SR, Khoury JC, Kalkwarf HJ. Human milk pumping rates of mothers of singletons and mothers of multiples. J Hum Lact. 2005;21:413-20.

32. Justesen A, Kunst A. Postneonatal and child mortality among twins in Southern and Eastern Africa. Int $\mathrm{J}$ Epidemiol. 2000;29:678-83.

33. Kaneko A, Kaneita Y, Yokoyama E, Miyake T, Harano S, Suzuki $\mathrm{K}$, et al. Factors associated with exclusive breast-feeding in Japan: for activities to support child-rearing with breast-feeding. J Epidemiol. 2006;16:57-63.

34. Kavanaugh K, Mead L, Meier P, Mangurten HH. Getting enough: mothers' concerns about breastfeeding a preterm infant after discharge. J Obstet Gynecol Neonatal Nurs. 1995;24:23-32.

35. Lau C, Hurst N, Bums P, Schanler RJ. Interaction of stress and lactation differs between mothers of premature singletons and multiples. Adv Exp Med Biol. 2004;554:313-6.

36. Liang R, Gunn AJ, Gunn TR. Can preterm twins breast feed successfully? NZ Med J. 1997;110:209-12.

37. Thome M, Alder EM, Ramel A. A population-based study of exclusive breastfeeding in Icelandic women: is there a relationship with depressive symptoms and parenting stress? Int J Nurs Stud. 2006;43:11-20.

38. Yokoyama Y, Wada S, Sugimoto M, Katayama M, Saito M, Sono J. Breastfeeding rates among singletons, twins and triplets in Japan: a population-based study. Twin Res Hum Genet. 2006;9:298-302.

39. Wilton JM. Breast feeding multiples. In: Keith LG, Papiernik E, Keith DM, Luke B, editors. Multiple Pregnancy: epidemiology, gestation and perinatal outcome. New York: Taylor \& Francis; 1995. pp. 553-61.

40. Ooki S. Population-based database of multiples in childhood of Ishikawa prefecture, Japan. Twin Res Hum Genet. 2006;9:832-7.

41. Ooki S. Language development of Japanese twins in childhood based on maternal reports. Jpn J Health Hum Ecology. 2005;71:12-24.

42. Ooki S. Motor development of Japanese twins in childhood as reported by mothers. Environ Health Prev Med. 2006;11:55-64.

43. Ooki S, Yokoyama Y. Physical growth charts from birth to six years of age in Japanese twins. J Epidemiol. 2004;14:151-60.

44. Ooki S, Asaka A. Comparison of obstetric and birthweight characteristics between the two largest databases of Japanese twins measured in childhood. Twin Res Hum Genet. 2005;8:638.

45. Kato N. Reference birthweight range for multiple birth neonates in Japan. BMC Pregnancy Childbirth. 2004;4:2.

46. Mamiya U. Japan's maternal and child health handbook. Midwives Chron. 1990;103:314-5.

47. Takayanagi K, Iwasaki S, Yoshinaka Y. The role of the maternal and child health handbook system in reducing perinatal mortality in Japan. Clin Perform Qual Health Care. 1993;1:29-33.
48. Ooki S, Asaka A. Zygosity diagnosis in young twins by questionnaire for twins' mothers and twins' self-reports. Twin Res. 2004;7:5-12.

49. Geraghty SR, Khoury JC, Kalkwarf HJ. Comparison of feeding among multiple birth infants. Twin Res. 2004;7:542-7.

50. SAS Institute Inc. SAS/STAT user's guide (version 8). Cary: SAS Institute, Inc.; 1999

51. Ooki S. Nongenetic factors associated with human handedness and footedness in Japanese twin children. Environ Health Prev Med. 2006;11:304-12.

52. Addy HA. The breast-feeding of twins. J Trop Pediatr Environ Child Health. 1975;21:231-9.

53. Ford RP, Mitchell EA, Scragg R, Stewart AW, Taylor BJ, Allen EM. Factors adversely associated with breast feeding in New Zealand. J Paediatr Child Health. 1994;30:483-9.

54. Yokoyama Y, Ooki S. Breast-feeding and bottle-feeding of twins, triplets and higher order multiple births. Nippon Koshu Eisei Zasshi. 2004;51:969-74.

55. Czeszynska MB, Kowalik K. Multiple pregnancy: factors contributing to early infant's breast-feeding-own experience. Acta Genet Med Gemellol (Roma). 1998;47:191-6.

56. Damato EG, Dowling DA, Madigan EA, Thanattherakul C. Duration of breastfeeding for mothers of twins. J Obstet Gynecol Neonatal Nurs. 2005;34:201-9.

57. Damato EG, Dowling DA, Standing TS, Schuster SD. Explanation for cessation of breastfeeding in mothers of twins. J Hum Lact. 2005;21:296-304.

58. Flidel-Rimon O, Shinwell ES. Breast-feeding multiples. Multiple pregnancy: epidemiology, gestation, and perinatal outcome, 2nd edn. In: Blickstein I, Keith LG, editors. Multiple pregnancy: epidemiology, gestation, and perinatal outcome, 2nd edn. New York: Taylor \& Francis; 2005. pp. 726-33.

59. Robarge JP, Reynolds ZB, Groothuis JR. Increased child abuse in families with twins. Res Nurs Health. 1982;5:199-203.

60. Aarts C, Kylberg E, Hornell A, Hofvander Y, Gebre-Medhin M, Greiner T. How exclusive is exclusive breastfeeding? A comparison of data since birth with current status data. Int $\mathbf{J}$ Epidemiol. 2000;29:1041-6.

61. Labbok M, Krasovec K. Toward consistency in breastfeeding definitions. Stud Fam Plann. 1990;21:226-30.

62. Githens PB, Glass CA, Sloan FA, Entman SS. Maternal recall and medical records: an examination of events during pregnancy, childbirth, and early infancy. Birth. 1993;20:136-41.

63. Li R, Scanlon KS, Serdula MK. The validity and reliability of maternal recall of breastfeeding practice. Nutr Rev. 2005;63:10310.

64. Tomeo CA, Rich-Edwards JW, Michels KB, Berkey CS, Hunter DJ, Frazier AL, et al. Reproducibility and validity of maternal recall of pregnancy-related events. Epidemiology. 1999;10:7747.

65. Yawn BP, Suman VJ, Jacobsen SJ. Maternal recall of distant pregnancy events. J Clin Epidemiol. 1998;51:399-405. 